\title{
Continuous Multiscale
} Entanglement Renormalization Ansatz as Holographic SurfaceState Correspondence

\section{$\operatorname{AUTHOR}(\mathrm{S})$ :}

Miyaji, Masamichi; Numasawa, Tokiro; Shiba, Noburo; Takayanagi, Tadashi; Watanabe, Kento

\section{CITATION:}

Miyaji, Masamichi ...[et al]. Continuous Multiscale Entanglement Renormalization Ansatz as Holographic Surface-State Correspondence. Physical Review Letters 2015, 115(17): 171602.

\section{ISSUE DATE:}

2015-10-22

URL:

http://hdl.handle.net/2433/252425

\section{RIGHT:}

(c) 2015 American Physical Society; 許諾条件に基づいて掲載しています 。 


\title{
Continuous Multiscale Entanglement Renormalization Ansatz as Holographic Surface-State Correspondence
}

\author{
Masamichi Miyaji, ${ }^{1}$ Tokiro Numasawa, ${ }^{1}$ Noburo Shiba, ${ }^{1}$ Tadashi Takayanagi, ${ }^{1,2}$ and Kento Watanabe ${ }^{1}$ \\ ${ }^{1}$ Yukawa Institute for Theoretical Physics, Kyoto University, Kitashirakawa Oiwakecho, Sakyo-ku, Kyoto 606-8502, Japan \\ ${ }^{2}$ Kavli Institute for the Physics and Mathematics of the Universe, University of Tokyo, Kashiwa, Chiba 277-8582, Japan
}

(Received 1 July 2015; revised manuscript received 7 August 2015; published 22 October 2015)

\begin{abstract}
We present how the surface-state correspondence, conjectured by Miyaji and Takayanagi, works in the setup of $\mathrm{AdS}_{3} / \mathrm{CFT}_{2}$ by generalizing the formulation of a continuous multiscale entanglement renormalization group ansatz. The boundary states in conformal field theories play a crucial role in our formulation and the bulk diffeomorphism is naturally taken into account. We give an identification of bulk local operators which reproduces correct scalar field solutions on $\mathrm{AdS}_{3}$ and bulk scalar propagators. We also calculate the information metric for a locally excited state and show that it reproduces the time slice of $\mathrm{AdS}_{3}$.
\end{abstract}

DOI: 10.1103/PhysRevLett.115.171602

PACS numbers: 11.25.Hf, 04.60.Cf, 11.25.Tq

Even though the idea of AdS/CFT correspondence [1] has led to tremendous progress in string theory, we still do not fully know its basic mechanism. It is obvious that the AdS/CFT correspondence is regarded as a special example of the holographic principle [2]; however, our current understandings of the holographic principle are far from complete.

The recently proposed duality called surface-state correspondence [3] gives a more detailed structure of the holographic principle. This duality can, in principle, be applied to any spacetimes described by Einstein gravity and even to those without timelike boundaries. This surfacestate correspondence (or simply called SS duality) argues a correspondence between a codimension two convex surface $\Sigma$ and a quantum state described by a density matrix $\rho(\Sigma)$, which is defined in the Hilbert space of quantum theory dual to the Einstein gravity. When this surface is closed and topologically trivial, the state is given by a pure state $\rho(\Sigma)=|\Sigma\rangle\langle\Sigma|$. In particular, if we consider Einstein gravity in an AdS space and take $\Sigma$ to be a time slice of the AdS boundary, then $|\Phi(\Sigma)\rangle$ is simply given by the ground state $|0\rangle$ of the dual conformal field theory (CFT); refer to Fig. 1.

The SS duality was proposed based on the recently found connection between the AdS/CFT and the tensor networks. Such a relation has been first proposed in Ref. [4] for a multiscale entanglement renormalization ansatz (MERA) [5] and later developed in Ref. [6] for a continuous multiscale entanglement renormalization group ansatz (cMERA) [7]. See also, e.g., Refs. [8-11] for various refinements and limitations of the connection between AdS/CFT and tensor networks. In general, a tensor network describes a wave function of a quantum state as a network diagram which fills a discretized space. The state $|\Phi(\Sigma)\rangle$ dual to a convex closed surface $\Sigma$ is constructed by contracting the indices of tensors which are included in the region surrounded by $\Sigma$. For example, in the network found in Ref. [9] we can explicitly construct the state $|\Phi(\Sigma)\rangle$ consistently by the above procedure. If the tensor network describes correctly a CFT ground state, then we expect the space described by the network is identical to a hyperbolic space. We would like to argue that the most direct way to realize tensor networks for CFTs is to employ the cMERA as we do not need to worry about lattice artifacts. It is also important that the Hilbert space structure is not changed under any smooth deformation of $\Sigma$ in the SS duality as we can always insert a dummy trivial state to keep the total dimension of Hilbert space the same.

The most elementary object in SS duality is the quantum state dual to a zero size closed surface, i.e., just a point. Such a state dual to a point in a gravitational spacetime is identified with the boundary state $|B\rangle$ [3]. This is because there is no real space entanglement for the state dual to such a pointlike surface, according to the idea of holographic entanglement entropy [12], and because the state with a vanishing real space entanglement entropy is given by the boundary state [13].

The latter fact can be naturally understood by turning off a relevant (e.g., mass) operator in a CFT suddenly at the (Euclidean) time $\tau=0$ as in the analysis of quantum quenches [14]. In terms of quantum states we find that

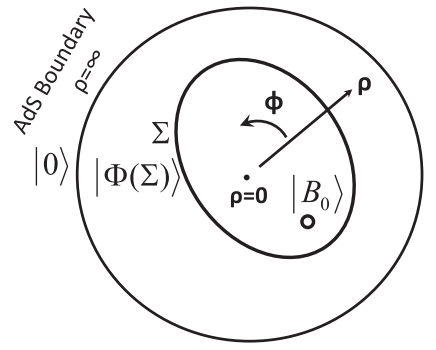

FIG. 1. A sketch of surface-state correspondence in AdS/CFT. 
the ground state $|0\rangle$ appears only for $\tau>0$ and thus it is equivalent to putting a sharp boundary at $\tau=0$ and restricting the spacetime to the region $\tau>0$. In 2D CFTs, such a physical boundary state is called a Cardy states $\left|C_{\alpha}\right\rangle$ [15], where $\alpha$ labels the primary fields $\Psi_{\alpha}$. An Ishibashi state $\left|I_{\alpha}\right\rangle$ [16] is a boundary state which includes only one sector of primary field $\Psi_{\alpha}$ and its descendants. A Cardy state is given by a specific linear combination of Ishibashi states.

Let us start with a cMERA description of the CFT ground state $|0\rangle$. We will employ the rescaled formalism in Ref. [6], which is obtained from the original construction [7] by getting rid of the rescaling procedure and which has an advantage that the Hilbert space does not change even if we consider a CFT on a compact manifold. The cMERA formulation is defined by a flow from the UV state given by the CFT vacuum $|0\rangle$ to the IR state, which has no real space entanglement.

As we have explained, we can identify such a state with one of the boundary states [13], denoted by $\left|B_{0}\right\rangle$. In $\mathrm{CFT}_{d+1}$ for $d \geq 3$, there is a conformal mass term in the gauge theory on $R \times S^{d}$ dual to a global $\mathrm{AdS}_{d+2}$. Moreover, the fundamental fields correspond to the internal directions such as $S^{5}$ in $\operatorname{AdS}_{5} \times S^{5}$ related to the $R$-symmetric directions. Therefore, it is natural to identify $\left|B_{0}\right\rangle$ with the boundary state for the Dirichlet boundary condition, which preserves the $R$ symmety. However, in a 2D CFT on a cylinder, there is no conformal mass and thus it is subtle whether $\left|B_{0}\right\rangle$ is a Cardy state or Ishibashi state. Nevertheless, to preserve the $R$ symmetry and other internal symmetries suggests that the IR state should be the Ishibashi state $\left|I_{0}\right\rangle$ for the identity sector. This is because in typical $\mathrm{AdS}_{3} / \mathrm{CFT}_{2}$ examples, the fundamental fields correspond to the other internal direction such as $T^{4}$ in $\mathrm{AdS}_{3} \times S^{3} \times T^{4}$ and we cannot impose any Dirichlet boundary condition for these fields without breaking $R$ symmetries and other global symmetries.

In this way, the cMERA is formulated as follows:

$$
|0\rangle=\mathcal{P} \exp \left(-i \int_{-\infty}^{0} d u \hat{K}(u)\right)\left|I_{0}\right\rangle,
$$

where $\hat{K}(u)$ is the disentangling operator at scale $u$ and $\mathcal{P}$ denotes the path ordering. The operation $\hat{K}(u)$ eliminates quantum entanglement longer than the length scale $\epsilon e^{-u}$, where $\epsilon$ is the UV cutoff or the lattice constant. The UV and IR limit corresponds to $u=0$ and $u=-\infty$, respectively. In other words, the disentangling operator takes the form $\hat{K}(u)=\int d k^{d} \Gamma\left(k e^{-u} \epsilon\right) O_{k}$, where $O_{k}$ is a disentangling operator with momentum $k$ and $\Gamma(x)=1-\theta(x)$ is a cutoff function $[\theta(x)$ is the Heaviside step function].

Once $\hat{K}(u)$ is given, the state $|0(u)\rangle$ at scale $u$ is defined:

$$
|0(u)\rangle=\mathcal{P} \exp \left(-i \int_{-\infty}^{u} d u \hat{K}(u)\right)\left|I_{0}\right\rangle .
$$

Thus, the cMERA is a unitary transformation (or a generalization of the Bogoliubov transformation) from the vacuum to the Ishibashi state. As $u$ increases, some quantum entanglement is added by the $\hat{K}(u)$ operation.

In AdS/CFT, we expect that the cMERA network describes the time slice of AdS space, i.e., hyperbolic space. Consider a 2D holographic CFT on a cylinder, whose coordinate is given by $(t, \phi)$ with the periodicity $\phi \sim \phi+2 \pi$. The dual $\mathrm{AdS}_{3}$ is given by the global coordinate

$$
d s^{2}=R^{2}\left(-\cosh ^{2} \rho d t^{2}+d \rho^{2}+\sinh ^{2} \rho d \phi^{2}\right) .
$$

In SS duality, we can consider the surface $\Sigma(u)$ dual to $|0(u)\rangle$. This surface coincides with a time slice of the AdS boundary for $u=0$ and shrink as $u$ decreases. Eventually, at $u \rightarrow-\infty$, it degenerates to a point at the origin of the AdS space. Therefore, we have $|0(0)\rangle=|0\rangle$ and $|0(-\infty)\rangle=\left|I_{0}\right\rangle$ as in Fig. 1. In the $\operatorname{AdS}_{3}$ coordinate (3), the IR limit $u=-\infty$ corresponds to the center $\rho=0$, while the UV limit $u=0$ corresponds to the AdS boundary $\rho \rightarrow \infty$.

The isometry of $\mathrm{AdS}_{3}$ is given by $S L(2, R)_{L} \times S L(2, R)_{R}$, which are generated by $\left(L_{1}, L_{0}, L_{-1}\right)$ and $\left(\tilde{L}_{1}, \tilde{L}_{0}, \tilde{L}_{-1}\right)$ dual to the (global) Virasoro symmetry of $2 d$ CFT. These are explicitly given by the following action in $\mathrm{AdS}_{3}$ [17]:

$$
\begin{aligned}
L_{0} & =i \partial_{+}, \quad \tilde{L}_{0}=i \partial_{-}, \\
L_{ \pm 1} & =i e^{ \pm i x^{+}}\left[\frac{\cosh 2 \rho}{\sinh 2 \rho} \partial_{+}-\frac{1}{\sinh 2 \rho} \partial_{-} \mp \frac{i}{2} \partial_{\rho}\right], \\
\tilde{L}_{ \pm 1} & =i e^{ \pm i x^{-}}\left[\frac{\cosh 2 \rho}{\sinh 2 \rho} \partial_{-}-\frac{1}{\sinh 2 \rho} \partial_{+} \mp \frac{i}{2} \partial_{\rho}\right] .
\end{aligned}
$$

In particular, we are interested in a $S L(2, R)$ subgroup of $S L(2, R)_{L} \times S L(2, R)_{R}$ which does not change the time slice $t=0$. It is generated by $\left(l_{1}, l_{0}, l_{-1}\right)$ defined by

$$
\begin{aligned}
l_{0} & =L_{0}-\tilde{L}_{0}=i \partial_{\phi}, \\
l_{-1} & =\tilde{L}_{1}-L_{-1}=i e^{-i \phi}\left[-\frac{1+\cosh (2 \rho)}{\sinh (2 \rho)} \partial_{\phi}-i \partial_{\rho}\right], \\
l_{1} & =\tilde{L}_{-1}-L_{1}=-i e^{i \phi}\left[\frac{1+\cosh (2 \rho)}{\sinh (2 \rho)} \partial_{\phi}-i \partial_{\rho}\right],
\end{aligned}
$$

which satisfy the $S L(2, R)$ algebra as usual, dual to Killing symmetry of the hyperbolic space $\mathrm{H}_{2}$.

The $S L(2, R)$ transformation $g(\rho, \phi)$ which takes the origin $\rho=0$ to a point $(\rho, \phi)$ on $\mathrm{H}_{2}$ is given by

$$
g(\rho, \phi)=e^{i \phi l_{0}} e^{(\rho / 2)\left(l_{1}-l_{-1}\right)} .
$$

It is obvious that the CFT vacuum $|0\rangle$ is invariant under this $S L(2, R)$ transformation. Moreover, boundary states have the same invariance, 


$$
g(\rho, \phi)\left|I_{\alpha}\right\rangle=\left|I_{\alpha}\right\rangle,
$$

which comes from the basic property $\left(L_{n}-\tilde{L}_{-n}\right)\left|I_{\alpha}\right\rangle=0$ of the boundary states. Thus the quantum states dual to points on the $\mathrm{H}_{2}$ are all given by the same state $\left|I_{0}\right\rangle$. This agrees with the argument of SS duality where all states whose dual surfaces are related by isometry are the same [3]. This is also consistent with the tensor network picture because this quantum state corresponds to the pointlike state in the network with no entanglement.

By acting $g(\rho, \phi)$ transformation, we can rewrite Eq. (1) as

$$
|0\rangle=\mathcal{P} \exp \left(-i \int_{-\infty}^{0} d u \hat{K}_{(\rho, \phi)}(u)\right)\left|I_{0}\right\rangle,
$$

where we defined $\hat{K}_{(\rho, \phi)}(u)=g(\rho, \phi) \cdot \hat{K}(u) \cdot g(\rho, \phi)^{-1}$. This transformation relates two different cMERA networks related by the conformal transformation by changing the choices of $u$ slices $\Sigma(u)$. We introduce

$$
U_{(\rho, \phi)}=\mathcal{P} \exp \left(-i \int_{-\infty}^{0} d u \hat{K}_{(\rho, \phi)}(u)\right)
$$

We would also like to mention one more important observation. Since boundary states preserve $l_{n}=\tilde{L}_{-n}-L_{n}$ even for $|n| \geq 2$, we can generalize $\hat{K}_{(\rho, \phi)}(u)$ into

$$
\hat{K}_{h}(u)=\hat{h}(u) \hat{K}(u) \hat{h}(u)^{-1}+i \partial_{u} \hat{h}(u) \cdot \hat{h}(u),
$$

where $\hat{h}(u)=\exp \left[\sum_{n} h_{n}(u) l_{n}\right]$. This transformation (10) is interpreted as the deformation of the intermediate surface $\Sigma_{u}$ dual to the state

$$
\left|\Phi\left(\Sigma_{u}\right)\right\rangle=\mathcal{P} \exp \left(-i \int_{-\infty}^{u} d u \hat{K}_{g}(u)\right)\left|I_{0}\right\rangle,
$$

which allows us to choose any possible foliation $\left\{\Sigma_{u}\right\}_{-\infty<u<0}$ of the time slice $H_{2}$. Note that as long as we assume $h_{n}(0)=0$, we always end with up the vacuum state $\left|\Phi\left(\Sigma_{0}\right)\right\rangle=|0\rangle$ at $u=0$. This confirms the proposed surface-state correspondence and we find that the diffeomorphism gauge symmetry that preserves the time slice is included in our generalized cMERA formulation.

Now we would like to turn to excitations by bulk fields. We insert a bulk quantized field $\hat{\psi}_{\alpha}(\rho, \phi)$ dual to a CFT primary field $\Psi_{\alpha}$, on the time slice $H_{2}$ at $(\rho, \phi)$. We argue that the locally excited state $\hat{\psi}_{\alpha}(\rho, \phi)|0\rangle_{\text {bulk }}$ is dual to the following CFT excited state $\left|\Psi_{\alpha}(\rho, \phi)\right\rangle$ :

$$
\begin{aligned}
\hat{\psi}_{\alpha}(\rho, \phi)|0\rangle_{\text {bulk }} & \in H_{\text {bulk }} \\
\leftrightarrow\left|\Psi_{\alpha}(\rho, \phi)\right\rangle=U_{(\rho, \phi)}\left|I_{\alpha}\right\rangle & \in H_{\mathrm{CFT}} .
\end{aligned}
$$

Since the metric should not change for the locally excited state except for the localized region, the state should have almost vanishing real space entanglement. Therefore, the IR state of the cMERA for the excited state is also given by a boundary state. Thus we can argue that the IR state is given by the Ishibashi state $\left|I_{\alpha}\right\rangle$ as in Eq. (12).

By taking this into the time evolution, we identify

$$
\hat{\psi}_{\alpha}(\rho, \phi, t) \leftrightarrow e^{i\left(L_{0}+\tilde{L}_{0}\right) t} U_{(\rho, \phi)} M_{\alpha} U_{(\rho, \phi)}^{-1} e^{-i\left(L_{0}+\tilde{L}_{0}\right) t},
$$

where $M_{\alpha}$ is an unitary operation which maps $\left|I_{0}\right\rangle$ to $\left|I_{\alpha}\right\rangle$. Refer to, e.g., Ref. [18] for an earlier standard literature of bulk field construction from CFT operators. We are taking a different route for the same purpose, as we are restricting on a specific time slice.

Next we want to identify the states $\left|\Psi_{\alpha}(\rho, \phi)\right\rangle$. For convenience, let us define $\left|\Psi_{\alpha}\right\rangle \equiv\left|\Psi_{\alpha}(0,0)\right\rangle$, such that $\left|\Psi_{\alpha}(\rho, \phi)\right\rangle=g(\rho, \phi)\left|\Psi_{\alpha}\right\rangle$. Remember that $\left|\Psi_{\alpha}\right\rangle$ is a CFT excited state dual to a local excitation in the bulk AdS at the origin $\rho=0$ at $t=0$. Thus its bulk geometry is excited only at the origin.

The $S L(2, R)$ subgroup of the original $S L(2, R)_{L} \times$ $S L(2, R)_{R}$, which preserves the point $\rho=t=0$, is not the same as the one generated by $l_{0}, l_{ \pm}$, but it is generated by $L_{0}-\tilde{L}_{0}, L_{1}+\tilde{L}_{-1}$, and $L_{-1}+\tilde{L}_{1}$, as can be confirmed from Eq. (4). Therefore, $\left|\Psi_{\alpha}\right\rangle$ should satisfy

$$
\left(L_{0}-\tilde{L}_{0}\right)\left|\Psi_{\alpha}\right\rangle=\left(L_{ \pm 1}+\tilde{L}_{\mp 1}\right)\left|\Psi_{\alpha}\right\rangle=0 .
$$

The simplest solution to this condition (13) is given by

$$
\left|\Psi_{\alpha}\right\rangle \propto e^{i(\pi / 2)\left(L_{0}+\tilde{L}_{0}\right)}\left|J_{\alpha}\right\rangle
$$

where $\left|J_{\alpha}\right\rangle$ is the "Ishibashi state" for the $S L(2, R)_{L} \times$ $S L(2, R)_{R}$ subalgebra of the Virasoro algebra. It is explicitly defined by $\left|J_{\alpha}\right\rangle=\sum_{k=0}^{\infty}|k\rangle_{L}|k\rangle_{R}$, where $|k\rangle_{L}$ (or $|k\rangle_{R}$ ) denotes the normalized (unit norm) descendant state proportional to $\left(L_{-1}\right)^{k}|\alpha\rangle$ (or $\left(\tilde{L}_{-1}\right)^{k}|\alpha\rangle$ ). We can confirm that this choice (14) is the correct state dual to the bulk local operator as we explain below. Note that if we, in particular, choose the primary state $|\alpha\rangle$ to be the vacuum $|0\rangle$, we find $\left|\Psi_{\alpha}(\rho, \phi)\right\rangle=|0\rangle$ as expected.

Indeed, by using the property (13), we can reproduce the correct scalar field solution on the $\mathrm{AdS}_{3}$. The bulk scalar field expectation value for the state $|\beta\rangle$ can be computed from the CFT inner product as follows

$$
\left\langle\hat{\psi}_{\alpha}(\rho, \phi, t)\right\rangle_{|\beta\rangle}=\left\langle\Psi_{\alpha}(\rho, \phi)\left|e^{-i t\left(L_{0}+\tilde{L}_{0}\right)}\right| \beta\right\rangle .
$$

By using the identity ( $\Delta_{\alpha}$ is the dimension of $\left.|\alpha\rangle\right)$ :

$$
\left\langle\Psi_{\alpha}\left|e^{-(\rho / 2)\left(l_{1}-l_{-1}\right)}\right| \alpha\right\rangle=\left\langle\Psi_{\alpha}\left|e^{\rho\left(L_{1}-L_{-1}\right)}\right| \alpha\right\rangle=\frac{1}{(\cosh \rho)^{2 \Delta_{\alpha}}},
$$


we can confirm that the scalar field expectation value for the primary state agrees with the known scalar field solution in $\mathrm{AdS}_{3}[17,19]$ as follows:

$$
\left\langle\hat{\psi}_{\alpha}(\rho, \phi, t)\right\rangle_{|\alpha\rangle} \propto e^{-2 i \Delta_{\alpha} t} \frac{1}{(\cosh \rho)^{2 \Delta_{\alpha}}} .
$$

It is also possible to extend the matching of Eq. (15) to $S L(2, R)$ descendants states. They are obtained by acting $L_{ \pm 1}$ and $\tilde{L}_{ \pm 1}$ on the primary state and the scalar field expectation values are obtained by acting the differential operators (4) on Eq. (16). These allow us to confirm the (perturbative) equation of motion for scalar fields,

$$
\left[L^{2}+\tilde{L}^{2}+m^{2} R^{2} / 2\right] \hat{\psi}_{\alpha}(t, \rho, \phi)=0,
$$

where $L^{2}=\left(L_{-1} L_{1}+L_{1} L_{-1}\right) / 2-L_{0}^{2}$ is the differential operator corresponding to the Casimir of $\operatorname{SL}(2, R)$ and we have $\Delta_{\alpha}=\frac{1}{2}+\frac{1}{2} \sqrt{m^{2} R^{2}+1}$. Moreover, we can prove that our inner product $\left\langle\Psi_{\alpha}(\rho, t, \phi) \mid \Psi_{\alpha}\left(\rho^{\prime}, t, \phi^{\prime}\right)\right\rangle$ in the 2D CFT perfectly matches with the known expression $[19,20]$ of bulk to bulk propagator of scalar fields.

In this way, we learned that the effect of the disentangling unitary transformation $U_{(\rho, \phi)}$ is to remove the (higher) Virasoro generators $L_{n}$ and $\tilde{L}_{n}$ with $|n| \geq 2$ and perform a time translation by $\pi / 2$.

Now, we can estimate how much the locality of bulk local operators persists when the separation $\left(\rho^{\prime}-\rho, \phi^{\prime}-\phi\right)$ gets larger by looking at the inner product $\left\langle I_{\alpha}\left|U_{(\rho, \phi)}^{-1} U_{\left(\rho^{\prime}, \phi^{\prime}\right)}\right| I_{\alpha}\right\rangle=\left\langle\Psi_{\alpha}(\rho, \phi) \mid \Psi_{\alpha}\left(\rho^{\prime}, \phi^{\prime}\right)\right\rangle$, where we used the symmetry (7). If this inner product is much smaller than 1, then we expect that bulk fields behave locally for that length scale as they look independent.

To see the behavior of the inner product, it is useful to employ the Fisher information metric $G_{a b}$ defined by

$$
\begin{aligned}
1 & -\left|\left\langle\Psi_{\alpha}(\rho, \phi) \mid \Psi_{\alpha}(\rho+d \rho, \phi+d \phi)\right\rangle\right| \\
& =G_{\rho \rho} d \rho^{2}+2 G_{\rho \phi} d \rho d \phi+G_{\phi \phi} d \phi^{2}+O\left(d \phi^{3}\right) .
\end{aligned}
$$

This measures the distance between the two states $\left|\Psi_{\alpha}(\rho, \phi)\right\rangle$ and $\left|\Psi_{\alpha}(\rho+d \rho, \phi+d \phi)\right\rangle$.

However, for our purpose, we do not want to have a literally delta functionally localized excitation, but want to smear over a length of the order of the Planck length. Indeed the state (14) is singular in that it has an infinite norm and thus we need a UV regularization. We expect that the energy of local excitation should not exceed the Planck energy. In AdS/CFT, the energy $E$ is related to the conformal dimension $\Delta$ via $E=\Delta / R$. If we substitute $E \ll 1 / l_{p} \sim 1 / G_{N}$, then we have the bound $\Delta \ll c$. Therefore, we would like to argue the identification,

$$
\left|\Psi_{\alpha}\right\rangle \propto e^{-\delta\left(L_{0}+\tilde{L}_{0}\right)} e^{i(\pi / 2)\left(L_{0}+\tilde{L}_{0}\right)}\left|J_{\alpha}\right\rangle
$$

where $\delta$ provided the UV cutoff and, therefore, we should take $\delta \sim(1 / c)$. Notice that even in the presence of this cutoff, we can maintain the basic properties of dual scalar field in AdS such as Eqs. (16) and (17), since we have $\delta \rightarrow 0$ in the large $c$ limit.

We evaluate the inner product up to quadratic terms

$$
\begin{aligned}
1 & -\left|\left\langle\Psi_{\alpha}(\rho, \phi) \mid \Psi_{\alpha}(\rho+d \rho, \phi+d \phi)\right\rangle\right| \\
& =\frac{1}{8}\left(d \rho^{2}+\sinh ^{2} \rho d \phi^{2}\right)\left\langle\Psi_{\alpha}\left|\left(l_{-1} l_{1}+l_{1} l_{-1}\right)\right| \Psi_{\alpha}\right\rangle .
\end{aligned}
$$

Here we employed Eq. (7) and the identity

$$
e^{-(\rho / 2)\left(l_{1}-l_{-1}\right)} l_{0} e^{(\rho / 2)\left(l_{1}-l_{-1}\right)}=\cosh \rho l_{0}-\sinh \rho \frac{l_{1}+l_{-1}}{2} .
$$

After some algebra, we obtain the estimation $\left\langle\Psi_{\alpha}\left|l_{-1} l_{1}\right| \Psi_{\alpha}\right\rangle \simeq 4\left\langle\Psi_{\alpha}\left|L_{-1} L_{1}\right| \Psi_{\alpha}\right\rangle \simeq\left(1 / 2 \delta^{2}\right)$. Thus, the information metric for $\left|\Psi_{\alpha}(\rho, \phi)\right\rangle$ is given by that of a hyperbolic space $\mathrm{H}_{2}$ :

$$
d s_{\mathrm{inf}}^{2}=\frac{1}{8 \delta^{2}}\left(d \rho^{2}+\sinh ^{2} \rho d \phi^{2}\right) .
$$

It is natural to expect that this corresponds to the time slice of the global $\mathrm{AdS}_{3}$, to which our 2D CFT is dual. Indeed, the radius of this $\mathrm{H}_{2}$ coincides [up to an $O(1)$ numerical factor] with that in the $\mathrm{AdS}_{3}$ metric with the Planck unit as we chose $\delta \sim 1 / c$.

Actually, it is not difficult to imagine how to get the full $\mathrm{AdS}_{3}$ metric including time components. As we argued, the two point function $\left\langle\Phi(\rho, t, \phi) \mid \Phi\left(\rho^{\prime}, t, \phi^{\prime}\right)\right\rangle$ coincides with the bulk to bulk propagator. Even more generally, when two points $X$ and $Y$ are close to each other, any two point function of a $d+1$ dimensional free scalar field gets proportional to $D(X, Y)^{-(d-1)}$, where $D(X, Y)$ is the geodesic distance between $X$ and $Y$. If we regularize this with a cutoff $\delta$, then we naturally have the normalized expression

$$
\langle\Phi(X) \mid \Phi(Y)\rangle \simeq \frac{\delta^{d-1}}{\left[D(X, Y)^{2}+\delta^{2}\right]^{d-1 / 2}} .
$$

This leads to the Fisher information metric $d s_{\text {inf }}^{2} \propto$ $\left(1 / \delta^{2}\right) g_{i j} d X^{i} d X^{j}$, where $g_{i j}$ is precisely the metric of the bulk spacetime. Since it is natural to choose $\delta$ to be the Planck scale, the information metric $d s_{\text {inf }}^{2}$ coincides with the bulk metric in the Planck unit.

In this Letter, we gave an explicit construction of the conjectured surface-state correspondence in the setup of $\mathrm{AdS}_{3} / \mathrm{CFT}_{2}$ by generalizing the formulation of cMERA. Our formalism naturally takes into account the bulk diffeomorphism as a gauge symmetry of cMERA formalism. We found an identification of bulk local operators that reproduces solutions of scalar field equations of motion on $\mathrm{AdS}_{3}$ and the bulk to bulk propagator. We also computed the information metric for a locally excited state and showed that it is given by that of a $2 \mathrm{D}$ hyperbolic manifold, which is argued to describe the time slice of $\mathrm{AdS}_{3}$. 
Even though it is an important future problem to find an analytical expression of disentangler $\hat{K}(u)$ for holographic CFTs, we can at least show how it looks schematically. It is natural to expect that in the UV region $u \simeq 0, \hat{K}(u)$ gets qualitatively similar to that for free CFTs, where $\hat{K}(u)$ is a bilinear of creation and annihilation operators of fundamental fields, which add $O(c)$ entanglement, as in Refs. [6,7]. On the other hand, we expect that in the IR region $|u| \ll 1$, the disentangler $\hat{K}(u)$ should be a linear combination of products of particular singlet operators (Virasoro operator s) as $\hat{K}(u) \sim \sum_{n} c_{n}(u) \Gamma\left(n e^{-u} \epsilon\right) L_{-n} \tilde{L}_{-n}$, which add only $O(1)$ entanglement in the IR, motivated by the confinement-deconfinement transition in holographic CFTs. It is curious to note that this IR behavior owing to the large $c$ limit may suggest cMERA can have a sub-AdS scale locality, as opposed to (discrete) MERA.

We thank John Cardy, Bartlomiej Czech, Sumit Das, Matthew Headrick, Sunil Mukhi, Masahiro Nozaki, Hirosi Ooguri, Xiao-Liang Qi, Shinsei Ryu, James Sully, Erik Tonni, Sandip Trivedi, Herman Verlinde, Guifre Vidal, and Beni Yoshida for useful conversations. We are grateful to the organizers and participants of the "International Workshop on Condensed Matter Physics and AdS/CFT" held in Kavli IPMU, Tokyo U. and the conference "Closing the entanglement gap" held in KITP, UCSB, where this work has been completed. T. N., N.S., and K. W. are supported by JSPS fellowships. T. T. is supported by JSPS Grant-in-Aid for Scientific Research (B) No. 25287058. T. T. is also supported by the World Premier International Research Center Initiative (WPI Initiative) from the Japan Ministry of Education, Culture, Sports, Science and Technology (MEXT).

Note added-After we finished this work, we noticed the interesting paper [21], which gave an identification of bulk local operator using boundary states in a different way.
[1] J. M. Maldacena, Adv. Theor. Math. Phys. 2, 231 (1998); Int. J. Theor. Phys. 38, 1113 (1999).

[2] G. 't Hooft, Salamfest (World Scientific, Singapore, 1993), p. 284; L. Susskind, J. Math. Phys. 36, 6377 (1995); D. Bigatti and L. Susskind, arXiv:hep-th/0002044.

[3] M. Miyaji and T. Takayanagi, Prog. Theor. Exp. Phys. 2015, 073B03 (2015).

[4] B. Swingle, Phys. Rev. D 86, 065007 (2012).

[5] G. Vidal, Phys. Rev. Lett. 99, 220405 (2007).

[6] M. Nozaki, S. Ryu, and T. Takayanagi, J. High Energy Phys. 10 (2012) 193.

[7] J. Haegeman, T. J. Osborne, H. Verschelde, and F. Verstraete, Phys. Rev. Lett. 110, 100402 (2013).

[8] X.-L. Qi, arXiv:1309.6282.

[9] F. Pastawski, B. Yoshida, D. Harlow, and J. Preskill, J. High Energy Phys. 06 (2015) 149.

[10] N. Bao, C. Cao, S. M. Carroll, A. Chatwin-Davies, N. Hunter-Jones, J. Pollack, and G. N. Remmen, Phys. Rev. D 91, 125036 (2015).

[11] B. Czech, L. Lamprou, S. McCandlish, and J. Sully, arXiv:1505.05515.

[12] S. Ryu and T. Takayanagi, Phys. Rev. Lett. 96, 181602 (2006); J. High Energy Phys. 08 (2006) 045.

[13] M. Miyaji, S. Ryu, T. Takayanagi, and X. Wen, J. High Energy Phys. 05 (2015) 152.

[14] P. Calabrese and J.L. Cardy, J. Stat. Mech. (2005) P04010.

[15] J. L. Cardy, Nucl. Phys. B324, 581 (1989).

[16] N. Ishibashi, Mod. Phys. Lett. A 04, 251 (1989).

[17] J. M. Maldacena and A. Strominger, J. High Energy Phys. 12 (1998) 005.

[18] D. Kabat, G. Lifschytz, and D. A. Lowe, Phys. Rev. D 83, 106009 (2011); I. Heemskerk, D. Marolf, J. Polchinski, and J. Sully, J. High Energy Phys. 10 (2012) 165; D. Kabat and G. Lifschytz, J. High Energy Phys. 09 (2015) 059.

[19] D. E. Berenstein, R. Corrado, W. Fischler, and J.M. Maldacena, Phys. Rev. D 59, 105023 (1999).

[20] U. H. Danielsson, E. Keski-Vakkuri, and M. Kruczenski, J. High Energy Phys. 01 (1999) 002.

[21] H. Verlinde, arXiv:1505.05069. 